\title{
Diagnosis, prevention, and management of canine hip dysplasia: a review
}

\author{
This article was published in the following Dove Press journal: \\ Veterinary Medicine: Research and Reports \\ 19 May 2015 \\ Number of times this article has been viewed
}

\author{
Emma R Schachner \\ Mandi J Lopez \\ Department of Veterinary Clinical \\ Sciences, School of Veterinary \\ Medicine, Louisiana State \\ University, Baton Rouge, LA, USA
}

Correspondence: Mandi J Lopez Department of Veterinary Clinical Sciences, School of Veterinary Medicine, Louisiana State University, Skip Bertman Drive, Baton Rouge, LA 70803, USA

Tel +l 2255789918

Email mlopez@lsu.edu
Abstract: Canine hip dysplasia (CHD) is a polygenic and multifactorial developmental disorder characterized by coxofemoral (hip) joint laxity, degeneration, and osteoarthritis (OA). Current diagnostic techniques are largely subjective measures of joint conformation performed at different stages of development. Recently, measures on three-dimensional images generated from computed tomography scans predicted the development of OA associated with CHD. Continued refinement of similar imaging methods may improve diagnostic imaging techniques to identify dogs predisposed to degenerative hip joint changes. By current consensus, joint changes consistent with CHD are influenced by genetic predisposition as well as environmental and biomechanical factors; however, despite decades of work, the relative contributions of each to the development and extent of CHD signs remain elusive. Similarly, despite considerable effort to decipher the genetic underpinnings of CHD for selective breeding programs, relevant genetic loci remain equivocal. As such, prevention of CHD within domestic canine populations is marginally successful. Conservative management is often employed to manage signs of CHD, with lifelong maintenance of body mass as one of the most promising methods. Surgical intervention is often employed to prevent joint changes or restore joint function, but there are no gold standards for either goal. To date, all CHD phenotypes are considered as a single entity in spite of recognized differences in expression and response to environmental conditions and treatment. Identification of distinct CHD phenotypes and targeting evidence-based conservative and invasive treatments for each may significantly advance prevention and management of a prevalent, debilitating condition in canine companions.

Keywords: canine hip dysplasia, orthopedics, joint, osteoarthritis

\section{Introduction}

Canine hip dysplasia (CHD) is a complex developmental disorder characterized by joint laxity and osteoarthritis (OA) in one or both coxofemoral (hip) joints (Figure 1A-C). ${ }^{1}$ The polygenic, multifactorial etiology ${ }^{2}$ of $\mathrm{CHD}$ has challenged veterinarians and researchers since the condition was described in the 1930s. ${ }^{3}$ Joint changes characteristic of CHD are also associated with environmental factors such as nutrition, ${ }^{4-6}$ exercise, ${ }^{7}$ and the process of skeletal ossification. ${ }^{8,9}$ The condition affects essentially all breeds, with an estimated prevalence ranging from $1 \%$ to $80 \%$ according to the Orthopedic Foundation for Animals. It appears to occur at a relatively high rate in large-bodied and brachycephalic dogs as well as those with high body length to height ratios. ${ }^{10,11}$ The periodic appearance of OA in joints other than the coxofemoral joint ${ }^{12,13}$ has led some to propose systemic contributions to CHD expression. ${ }^{1}$ These complexities, among others, complicate attempts to manage the CHD by selective breeding despite strict reporting and guidelines. ${ }^{14}$ 

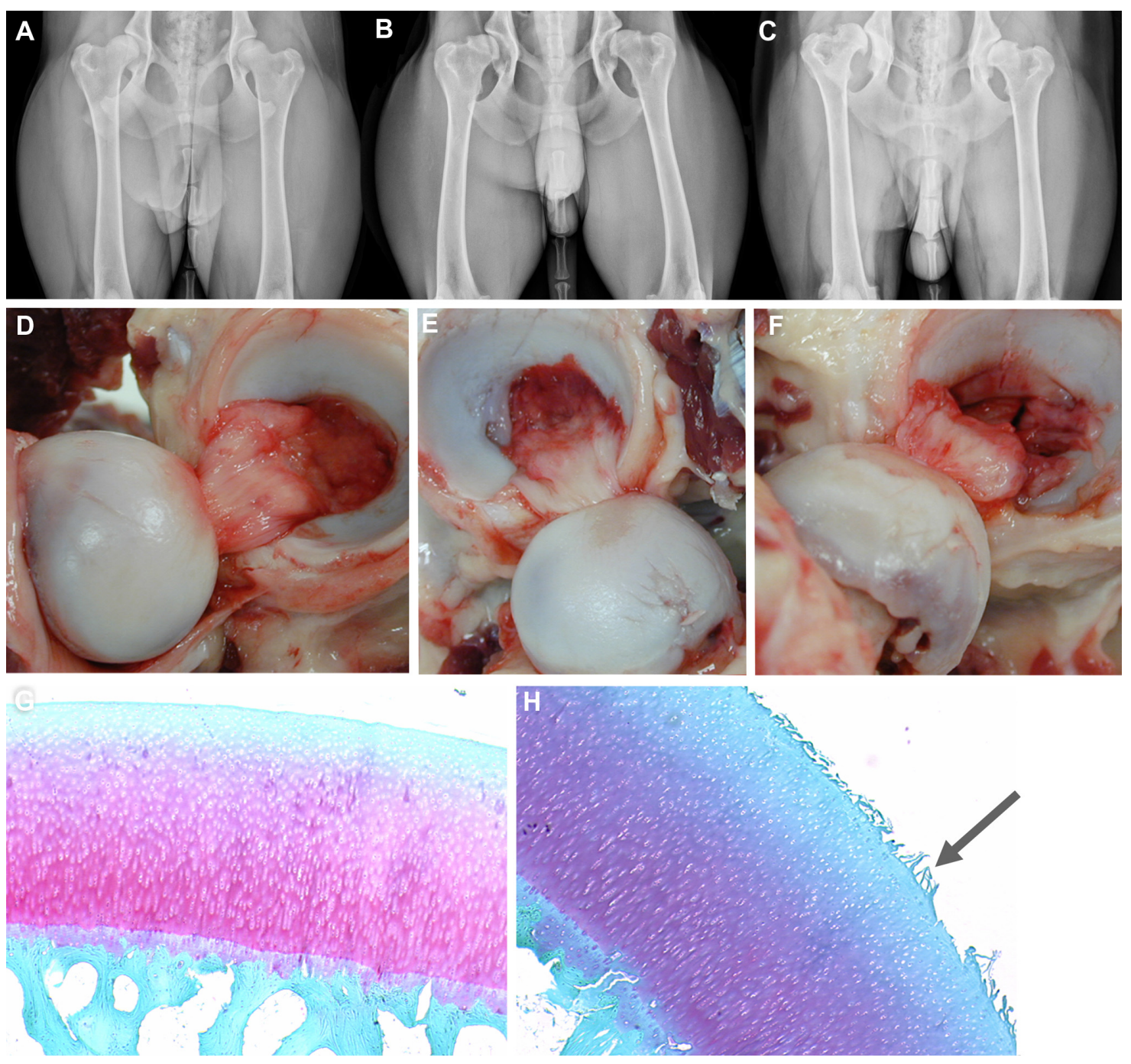

Figure I Anatomy of canine hip dysplasia.

Notes: (A-C) Canine hip-extended radiographs, and corresponding images of the joints (D-F) from different individuals demonstrating mild (A and $\mathbf{D})$, moderate (B and $\mathbf{E})$, and severe $(\mathbf{C}$ and $\mathbf{F})$ joint changes. Light photomicrographs of normal $(\mathbf{G})$ and fibrillated $(\mathbf{H}$, arrow) articular cartilage.

There are many theories to explain CHD joint degeneration, but joint laxity and irregular or delayed endochondral ossification are among the most popular. The conditions are not mutually exclusive, and their phenotypic expression is variable within and among breeds. ${ }^{15}$ Partially ossified hip structures may become distorted during development due to mechanical stresses in joints with delayed endochondral ossification. ${ }^{8,16}$ Joint components may be more vulnerable to deformation and damage from normal joint kinetics before they are fully ossified. ${ }^{8,917}$ Abnormal and delayed endochondral ossification in the coxofemoral joint has been identified in 15-day-old dogs that developed CHD by the time they were 12 months old, ${ }^{8,9,18}$ and in Great Danes with experimentally induced hip dysplasia. ${ }^{19}$ In contrast, comparably earlier joint ossification appears to occur in Greyhounds, a breed with one of the lowest incidences of CHD. While it is clear that variation in the process of endochondral ossification may play a role in the development of CHD, the exact relationships between ossification patterns, abnormal joint structure, and development of OA remain unclear. ${ }^{20}$

Affected joints usually develop varying degrees of synovial inflammation, articular cartilage damage (Figure 1), osteophytes, and subchondral bone sclerosis and remodeling. ${ }^{21-23}$ While there is no single, overarching description of the sequence of events in the process, there are changes that occur in many forms of dysplasia. Recently, the dorsal acetabular rim angle (a measure of the dorsal slope [angle] of the subchondral articular acetabular surface relative to horizontal) was reported to be significantly larger (less femoral head coverage by the acetabulum) in dogs with coxofemoral joint laxity versus normal dogs as early as 1 week of age. ${ }^{17}$ Subluxation of the femoral head and delays in ossification 
of the craniodorsal acetabular margin are often visible by 8 weeks, and, in many cases, subluxation of the femoral head increases by around 12 weeks of age. ${ }^{18}$ Degeneration and microfractures of the articular cartilage, and thickening, inflammation, and deterioration of the joint capsule, tendinous insertions, and ligaments are often apparent by 5 months of age.$^{18}$ Despite the presence of these degenerative traits in many dogs with degenerative coxofemoral joint changes, clinical signs are variable. ${ }^{21}$

A direct relationship between joint capsular collagen composition and mechanical properties was proposed over 30 years ago. ${ }^{24}$ Altered capsular collagen composition has been identified in children with congenitally dislocated hips $^{25}$ and dogs with hip joint laxity. ${ }^{26}$ Joint capsular collagen fibrils were found to be more heterogeneous in 8-month-old Labrador Retriever puppies with severe coxofemoral joint laxity than those with normal joints. ${ }^{15}$ Abnormal collagen composition is thought to contribute to reduced joint capsule stiffness, which contributes to excess femoral head motion and abnormal mechanical stresses on the femoral greater trochanter and acetabular margins and cartilage. ${ }^{15}$ Over time, the abnormal forces are thought to result in deformation of the articulating structures and an incongruous joint. ${ }^{18}$

Despite almost a century of work, many aspects of the development and progression of joint changes and OA associated with CHD remain elusive. This makes establishment of a gold standard for treatment a challenge. The lack of a single, predictable pattern of joint degeneration is likely a reflection of natural variability, including individual responses to external environmental influences. However, ambiguity in disease progression may also reflect distinct disease processes that have yet to be recognized. Continued efforts to identify and characterize patterns in joint changes may lead to identification of CHD phenotypes, which will, in turn, contribute to earlier disease identification and more effective targeted treatments.

\section{Diagnosis}

Despite some recognized patterns of joint degeneration characteristic of $\mathrm{CHD}$, there is significant variability in the progression and ultimate severity of the disease as well as inconsistent relationships between gross and radiographic joint changes and clinical signs. ${ }^{21}$ There are, however, two general behaviors often attributed to CHD, including lameness in young dogs (under 1 year), that increases with activity or trauma, and gait abnormalities and hind limb muscle atrophy in older dogs. ${ }^{27}$ Notably, hind limb lameness can be due to reasons other than CHD joint changes, including pelvic, distal hind limb, and neurological pathologies, metabolic bone disease, ligament rupture, patellar luxation, and spine disorders. ${ }^{27}$ Hence, a thorough, comprehensive assessment is paramount to identification of the source of discomfort.

\section{Subjective laxity examinations}

The Ortolani test is a subjective evaluation of coxofemoral joint laxity originally designed for diagnosis of human congenital hip dislocation in the $1930 \mathrm{~s} .{ }^{28,29}$ The test is also used as a CHD screening test. ${ }^{28}$ Dogs are placed in lateral recumbency; one hand of the examiner is used to apply force along the length of the femur from the stifle toward the pelvis as the other braces the back just above the sacrum (Figure 2). ${ }^{27}$ This maneuver is intended to displace the femoral head. The stifle is then slowly abducted to reduce the joint. ${ }^{29}$ An audible or palpable pop as the femur slips back into the acetabulum is considered a positive Ortolani sign indicative of joint laxity. Lack of an Ortolani sign does not necessarily mean that the hip is normal. Joint changes associated with dysplasia, like thickening of the joint capsule and joint tissue, may interfere with the displacement required for a positive sign. ${ }^{28,30}$ Bardens' test, ${ }^{31}$ an examination technique designed to evaluate the hips of babies (aged younger than 6 months), is thought to be more sensitive for detecting coxofemoral joint laxity and/or shallow acetabula in puppies 6-8 weeks of age. ${ }^{29}$ With the dog in lateral recumbency, the proximal femur is elevated laterally from the body. With the femur elevated, the index finger of the other hand is used to push the femur away from the joint in a dorsal direction with pressure on the greater trochanter. More than $2 \mathrm{~mm}$ of displacement is considered a positive sign. ${ }^{31}$ In general, these and other palpation techniques may be used as part of a comprehensive examination on puppies or dogs suspected to have excessive joint laxity characteristic of CHD. However, the tests alone are not sufficient for diagnosis of CHD.

\section{Radiography}

Radiography has long been the gold standard to assess and quantify joint changes associated with CHD joint remodeling. ${ }^{32,33}$ Worldwide, there are five popular, standardized evaluation systems with distinct metrics that are used to grade canine radiographic coxofemoral joint conformation and degenerative changes.

\section{Orthopedic Foundation for Animals}

The Orthopedic Foundation for Animals evaluation is performed on hip-extended radiographs performed under heavy sedation or general anesthesia by three independent board-certified radiologists. ${ }^{10}$ Based on subjective assessment of nine joint parameters (Figure 3A), conformation 

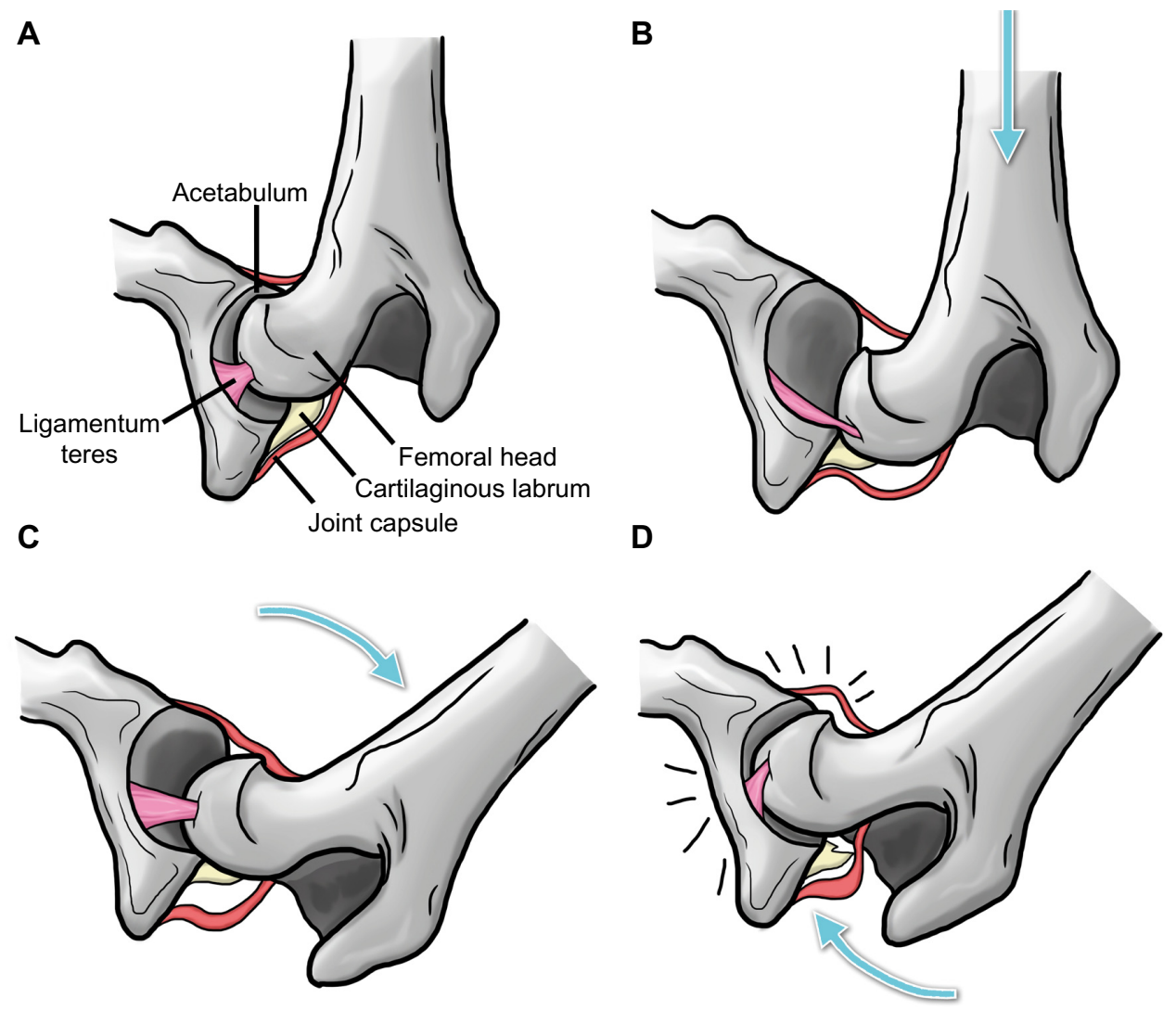

Figure 2 Schematic illustration of the Ortolani test.

Notes: Image demonstrates the coxofemoral joint prior to distraction $(\mathbf{A})$, while force is applied from the stifle toward the hip along the axis of the femur to displace the femoral head (B), during abduction of the femur to reduce the joint (C), and with the femoral head snapping back into place with an audible click, ie, the Ortolani sign (D). Arrows indicate the direction of the applied force. Adapted from Chalman JA, Butler HC. Coxofemoral joint laxity and the Ortolani sign. Journal of American Animal Hospital Association. 1985;21:671-676. ${ }^{28}$

is categorized as excellent, good, fair, borderline, mild, moderate, or severe. The first three categories are considered to be normal while the last three are dysplastic. ${ }^{10}$ Metrics are largely subjective assessments of hip conformation and evidence of degenerative joint disease.

\section{British Veterinary Association/Kennel Club}

The British Veterinary Association/Kennel Club maintains a "pass/fail" evaluation system that was instituted in 1965 and updated in $1984 .{ }^{34}$ For scoring, dogs must be at least 1 year of age, microchipped (or tattooed), and, if registered with the Kennel Club, the registration number must be included on the radiograph. ${ }^{35}$ Each dog has one opportunity to be scored by the system. Joints are individually scored on nine criteria from 0 to 5 or from 0 to 6 on hip-extended radiographs by two qualified radiologists on a British Veterinary Association/Kennel Club panel, with 0 being the best score and 106 the worst (53 possible points for each hip). ${ }^{34}$ The nine criteria (Figure 3B) include the Norberg angle (Figure 3C) and subjective assessments including subluxation, dorsal acetabular edge, cranial acetabular edge, cranial effective acetabular rim, acetabular fossa, femoral head recontouring, and femoral head and neck exostosis. ${ }^{34,35}$ An average score for each individual dog breed is published, ie, the breed mean score, and it is recommended that only animals with total scores well below the breed mean be used for breeding purposes..$^{35}$

\section{Fédération Cynologique Internationale}

The Fédération Cynologique Internationale $(\mathrm{FCI})^{36}$ is one of the largest canine organizations in the world and includes kennel clubs from across Europe, Asia, Africa, and South America. Extended hip and abducted hind limb radiographs performed at 1 year of age (18 months for large breed dogs) are scored according to the official FCI system by radiologists approved by breed-specific kennel clubs. ${ }^{36}$ Scoring includes the Norberg angle, formed by a horizontal line connecting the centers of the right and left femoral heads and a line connecting each center to the cranial margin of the corresponding acetabulum (Figure 3C) ${ }^{37}$ as well as subjective hip conformation parameters. Each joint is assigned a grade of A-E, with A representing healthy and $\mathrm{E}$ representing severe dysplasia. The more dysplastic of the two joint scores is considered the 
A
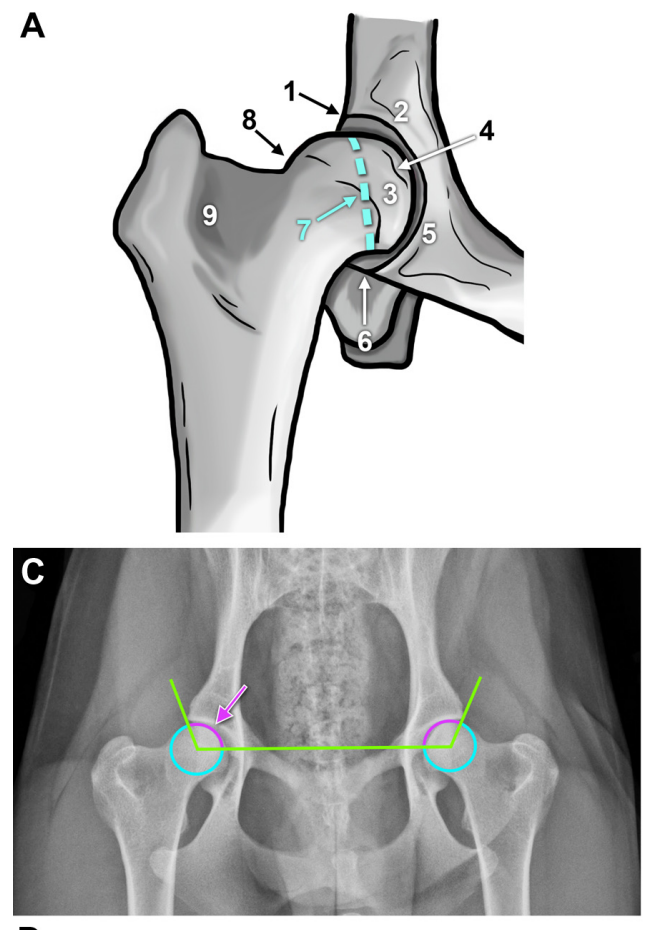

D

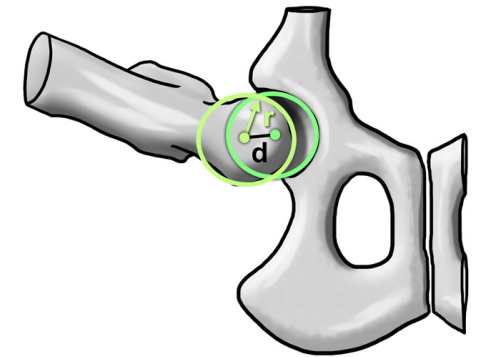

B
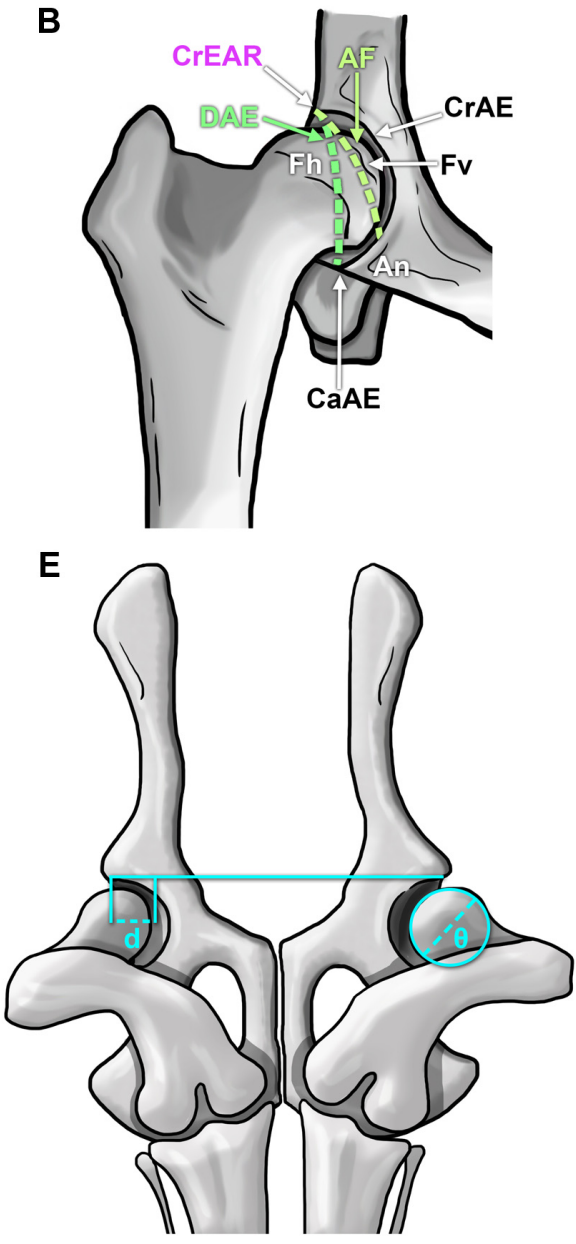

Figure 3 Representations of anatomical landmarks and evaluation mechanisms to assess canine hip dysplasia.

Notes: Coxofemoral joint anatomical characteristics considered by the Orthopedic Foundation for Animals (A): craniolateral acetabular rim (I), cranial acetabular margin (2), femoral head (3), fovea capitis (4), acetabular notch (5), caudal acetabular margin (6), dorsal acetabular margin (7), junction of femoral head and neck (8), and trochanteric fossa (9). (B) British Veterinary Association/Kennel Club canine coxofemoral joint characteristics scored during evaluation. ${ }^{10,34}$ Schematic superimposed on a hip-extended radiograph demonstrating the Norberg angle (C, arrow). Illustration of the Pennsylvania Hip Improvement Program (distraction index, the distance between the centers of the femoral head and acetabulum during distraction (D) divided by the radius ( $r$ ) of the femoral head (d). ${ }^{41}$ Depiction of the dorsolateral subluxation score (E) calculated as 100 multiplied by the percentage of femoral head medial to the cranial acetabular rim (d) divided by the femoral head diameter $(\theta)$, $d / \theta \times 100 \%)$.

Abbreviations: AF, acetabular fossa; An, acetabular notch; CaAE, caudal acetabular edge; CrAE, cranial acetabular edge; CrEAR, cranial effective acetabular rim; DAE, dorsal acetabular edge; Fh, femoral head; Fv, foveal defect.

final score for the individual dog. The same grading scale can also be applied to computed tomography (CT) scans.

\section{Pennsylvania Hip Improvement Program}

University of Pennsylvania researchers developed a quantitative method to evaluate canine hip conformation ${ }^{38-40}$ that was implemented in $1994 .{ }^{41}$ The primary distinction of the Pennsylvania Hip Improvement Program (PennHIP) method is that passive hip joint laxity is measured in addition to subjective radiographic conformation. ${ }^{38-40}$ Three radiographic views are evaluated by PennHIP-certified radiologists: a standard hip-extended view for evidence of degenerative joint disease; a compression view for congruity between the femoral head and acetabulum; and a distraction view, for joint laxity. The distraction index is the ratio of the distance between the centers of the femoral head and acetabulum (d) and the radius of the femoral head (r), as shown in Figure 3D. The closer the score is to 0 , the better the fit, ie, minimal femoral distraction, but a score of 1 indicates severe laxity and associated femoral distraction. ${ }^{41}$ Recently, the PennHIP distraction index and OA scores were found to have strong correlations with microstructural changes in the articular cartilage, ${ }^{42}$ potentially indicating a relationship between joint laxity measured by this technique and articular surface degeneration.

\section{Dorsolateral subluxation}

Dorsolateral subluxation is used to quantify joint laxity in a position to simulate weight-bearing (Figure 3E). During 
general anesthesia, pressure is applied to the femur at the level of the stifle while imaging the dog in ventral recumbency. ${ }^{43}$ Joints with less than $45 \%$ coverage of the femoral head by the lateral aspect of the cranial acetabular rim have an increased chance of developing joint changes and OA over time compared with those with a higher percentage $(>55 \%)$ of coverage. ${ }^{43}$

Subjective radiographic evaluations are limited by the inherent variability associated with examiners, image quality, and differences between animals including periarticular soft tissue changes and muscle atrophy. Variation in the degree of muscle relaxation associated with sedation or anesthesia during imaging can influence the ability to identify joint abnormalities by as much as $50 \%{ }^{44}$ Further, each evaluation system is distinct, so results are based on slightly different criteria. Recently, the Orthopedic Foundation for Animals score was reported to underestimate the likelihood of developing coxofemoral joint OA compared to the PennHIP distraction index. ${ }^{45}$ Reporting mechanisms also vary widely in public access to individual scores for reproduction decisions. As with any measure, radiographic hip scores should not be used in isolation to evaluate and predict current and future joint structure and function. It is possible that the presence of OA at a young age may be indicative of rapidly progressive joint disease, and, given recognition of the genetic basis for the disease, consideration of the presence and extent of CHD signs in related individuals is likely warranted. Based on this information, it is clear that continued efforts to identify mechanisms for early and accurate CHD diagnosis are of utmost importance. Adaptation of knowledge from decades of research to emerging imaging modalities will, no doubt, continue to improve upon current standards.

\section{Computed tomography}

CT technology for pelvic imaging has improved considerably over the past few decades. While radiographs remain the primary method used to image canine coxofemoral joints, CT is becoming increasingly popular. Using threedimensional CT models, a recent longitudinal study showed that volumetric changes in the acetabulum and proximal femur occurred in a predictable manner during skeletal growth in a colony of dogs with coxofemoral joint laxity. ${ }^{46}$ Another study demonstrated that two-dimensional CT images and three-dimensional models created from CT images can be used to predict the presence of joint OA at maturity. ${ }^{47}$ Two-dimensional CT measures included the percent femoral head coverage, acetabular index, and the following angles: acetabular anteversion, ventral, dorsal, and horizontal acetabular sector, center edge, and horizontal toit externe (Figure 4). Measures on three-dimensional models included femoral head and neck volumes, femoral head and neck radii and femoral neck angle (Figure 4). The 16-week distraction index and center edge angle combination was the best predictor of mature OA, whereas the 32-week dorsal acetabular sector angle and Norberg angle combination was the most effective predictor of the presence of OA at maturity. Hence, combined measures were the best mechanism for predicting development of OA, and the combinations varied with age. In a separate study, numerous measures were performed on pelvic CT scans of beagles and mixed breed dogs at various time points between the ages of 2 months and 1 year to assess the relationship of the measures with joint laxity. ${ }^{17}$ The dorsal acetabular rim angle and center distance index (the distance between the femoral head and the center of the acetabulum, divided by the radius of the femoral head, or the PennHIP ${ }^{41}$ distraction index) were found to be good indicators of joint laxity and dysplastic changes. ${ }^{17}$ Magnetic resonance imaging is used to evaluate the three-dimensional structure of human articular soft tissues, and relatively recently, canine articular soft tissues, ${ }^{48}$ but CT is best for bone structure, ${ }^{49}$ and the cost of magnetic resonance imaging for screening may be prohibitive. As technology advances, and CT and magnetic resonance imaging become more readily available and affordable, use of three-dimensional imaging methodologies will likely become an integral part of diagnosis and assessment of CHD.

\section{Therapeutic management and intervention Conservative management}

There are numerous descriptions of multifactorial systems, with numeric, visual analog, and descriptive scales to reproducibly evaluate joint pain associated with CHD. ${ }^{50}$ Many of the assessments within the systems are subjective evaluations of individual behavior or responses, and there is no single gold standard with which to quantify hip pain in the dog. ${ }^{50}$ While efforts continue to establish a uniform, standard evaluation system for canine hip joint pain, those systems that include multiple subjective and objective assessments by individuals who are not aware of specific treatments or conditions, are often informative.

Conservative management of CHD generally consists of a combination of mechanisms to reduce progression of joint damage and alleviate discomfort. ${ }^{51}$ Nonsteroidal antiinflammatory drugs are commonly used for pain associated 

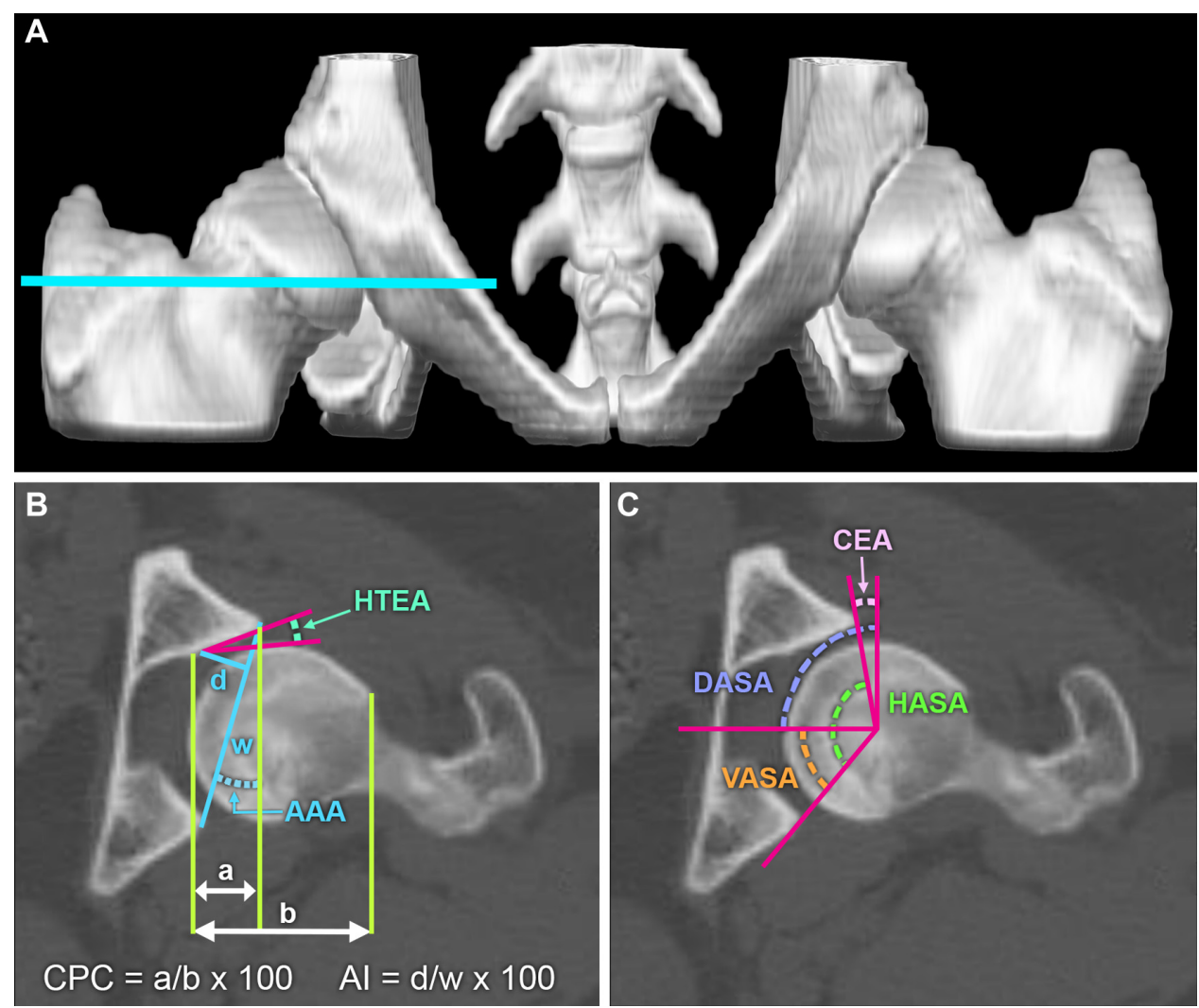

Figure 4 Measurements and three-dimensional models for evaluating the dysplastic canine hip.

Notes: (A) Volume rendered model of the canine pelvis generated from two-dimensional computed tomography images (B and $\mathbf{C})$. The blue line in (A) indicates the level of the cross-sectional image in (B) and (C). (B) and (C) Representative measures performed on two-dimensional computed tomographic images of the canine coxofemoral joint. Acetabular index is the ratio between the width and the depth of the acetabulum; $\mathrm{d} / \mathrm{w} \times 100$. For further information see Lopez et al ${ }^{42}$ and $\mathrm{Andronescu}$ et al. ${ }^{47}$ For details on these measures see Lopez et al. ${ }^{42}$

Abbreviations: AAA, acetabular anteversion angle; AI, acetabular index; CEA, center edge angle; CPC, percentage of femoral head coverage; DASA, dorsal acetabular sector angle (dorsal coverage of the femoral head); HASA, horizontal acetabular sector angle (total acetabular coverage of the femoral head); HTEA, horizontal toit externe angle (orientation of the acetabulum); VASA, ventral acetabular sector angle (ventral coverage of the femoral head).

with severely arthritic joints in dogs. ${ }^{52}$ Numerous studies indicate that achieving and maintaining a healthy body weight contributes to delayed onset and reduced clinical signs associated with hip joint pain., ${ }^{4,53}$ Various food supplements reported to alleviate signs of coxofemoral joint pain from $\mathrm{OA}$ range from green-lipped mussels (Perna canaliculus) ${ }^{54}$ to fish oil. ${ }^{55}$ Polysulfated glycosaminoglycan supplements and injections have been recommended for prevention and treatment of OA in dogs and other mammals. ${ }^{56-59}$ Intramuscular $^{57}$ and intra-articular administration has also been reported, ${ }^{56}$ but responses vary. ${ }^{51}$ Alternative methods that have also been investigated for the treatment of painful CHD joints include acupuncture and gold bead implantation, among others. The implantation of gold beads at acupuncture points was developed in the USA in the 1970s and implemented to a limited degree in veterinary medicine in the 1990s for degenerative joint disease pain..$^{60,61}$ Results are mixed, with some studies showing clinical improvement ${ }^{61,62}$ and others showing no discernible effect. ${ }^{63}$
Maintenance of optimum body weight has long been considered one of the most effective methods for reducing the signs associated with dysplasia and OA. ${ }^{4} \mathrm{~A}$ lifelong dietary restriction of $25 \%$ delayed the appearance of OA as well as the intensity of clinical signs in Labrador Retrievers compared with feeding ad libitum. ${ }^{53}$ Weight loss in conjunction with physiotherapy that included transcutaneous electrical nerve stimulation improved the clinical outcome for obese dogs with radiographic signs of OA.${ }^{64}$ Recently, intra-articular botulinum toxin $\mathrm{A}$ was reported to reduce the pain associated with OA based on improvements in limb use (ie, gait patterns) measured with a force platform. ${ }^{65}$ At present, there are few reports of long-term studies concerning the efficacy of nonsurgical or conservative treatment of $\mathrm{CHD}$ joint changes. These studies can be limited by the challenges of consistent monitoring and reporting by multiple and individual owners, as well as a wide range of disease severity and canine personalities. ${ }^{66}$ However, a recent retrospective report indicates that conservative and nonsurgical management (ie, weight 
control, reduced exercise, and analgesics) of 74 dogs over the span of 13 years did not improve quality of life as anticipated from previous reports. ${ }^{66}$

There is significant interest in the use of regenerative medicine to treat signs of $\mathrm{CHD}$ and $\mathrm{OA}$; however, much of the information reported is subjective in nature. Currently, numerous controlled, preclinical and clinical trials are underway that may provide some perspective on the value of this emerging technology. Intra-articular injection of adiposederived stem cells has been found to be a safe therapeutic approach for the treatment of symptoms associated with OA. ${ }^{67}$ Preliminary studies show that injection of adiposederived stem cells into affected joints may reduce clinical signs of hip pain (ie, lameness) based on subjective clinical evaluations ${ }^{67}$ and force platform gait analysis. ${ }^{68,69}$ A randomized comparison between a single intra-articular injection of adipose-derived stem cells or plasma rich in growth factors showed that both treatments reduced behavior associated with pain, but that the adipose-derived stem cells appeared to be more effective for up to 6 months post-treatment based upon owner assessments. ${ }^{70}$ This information clearly demonstrates that there is more work to be done on the efficacy of conservative and alternative methods to manage signs of CHD.

\section{Surgery}

Despite the prevalence of CHD, a gold standard surgical procedure has yet to be identified. ${ }^{71}$ As such, there are numerous surgeries to prevent progression of degenerative joint changes or alleviate pain and restore joint function.

Some surgical procedures designed to prevent onset of OA in hips identified as being predisposed to development of $\mathrm{OA}^{72}$ include double and triple pelvic osteotomy, acetabular shelf and excision arthroplasty, femoral osteotomy, and juvenile pubic symphysiodesis. ${ }^{73}$ Both juvenile pubic symphysiodesis and triple pelvic osteotomy are designed to increase femoral head coverage by ventrolateral rotation of the acetabulum. The juvenile pubic symphysiodesis procedure involves premature closure of the pubic symphysis. ${ }^{72,74}$ Resulting reduction in the pelvic inlet width causes ventrolateral rotation of the acetabulum during pelvic growth, and is thought to result in a 40\%-46\% improvement in acetabular and dorsal acetabular rim angles compared to no treatment. ${ }^{73-75}$ Juvenile pubic symphysiodesis appears to have the best outcomes when performed in puppies that are $12-16$ weeks old. ${ }^{73}$ Triple pelvic osteotomy is a much more extensive procedure, and involves osteotomies of the ilium, pubis, and ischium to allow manual rotation of the acetabulum for better femoral head coverage. ${ }^{76}$ The ilial osteotomy is stabilized with bone plates customized to accommodate the rotation. ${ }^{77}$ This procedure is generally recommended for young dogs without irreversible (or with mild) degeneration of the coxofemoral joint. ${ }^{77,78}$ Information about long-term outcomes of the various surgical treatments is limited. Preliminary reports indicate that juvenile pubic symphysiodesis and triple pelvic osteotomy minimally affect laxity and femoral head coverage when performed at 5 months or older ${ }^{72}$ compared with earlier reports indicating that the procedure performed slightly earlier (15 weeks) improved acetabular coverage of the femur. ${ }^{75}$ Mechanisms to reduce the OA characteristic of CHD will undoubtedly improve quality of life for affected dogs. Long-term outcomes will help identify treatments toward this end.

Total hip replacement (Figure 5) is often applied in advanced cases of joint degeneration and is considered a salvage procedure. ${ }^{79}$ There are no clear guidelines for the best time to implement total hip replacement, but the average time between onset of signs and surgery is 10 months. ${ }^{80}$ Total hip replacement procedures in dogs began in the 1970s, and a modular system was introduced in the mid 1990s that coupled a fixed monobloc cobalt-chromium alloy femoral implant with an acetabular cup for cemented fixation. ${ }^{81}$ Further refinements to total hip replacement implants have contributed to a high clinical recovery rate, with loosening of the acetabular cup and cup wear reported as some of the most common complications. ${ }^{81}$ More recently, cementless fixation has been developed, and is reported to have positive results. ${ }^{79}$ As the name implies, femoral and acetabular implants are cemented to bone for cemented total hip replacement. In contrast, cementless fixation or uncemented implants are designed so that the bone grows into or onto the prosthesis without the need for cement at the bone-implant interface. Implant loosening is reported to be less than for cemented implants. ${ }^{79,82}$ A primary concern associated with total hip replacement is the potential for an inflammatory response

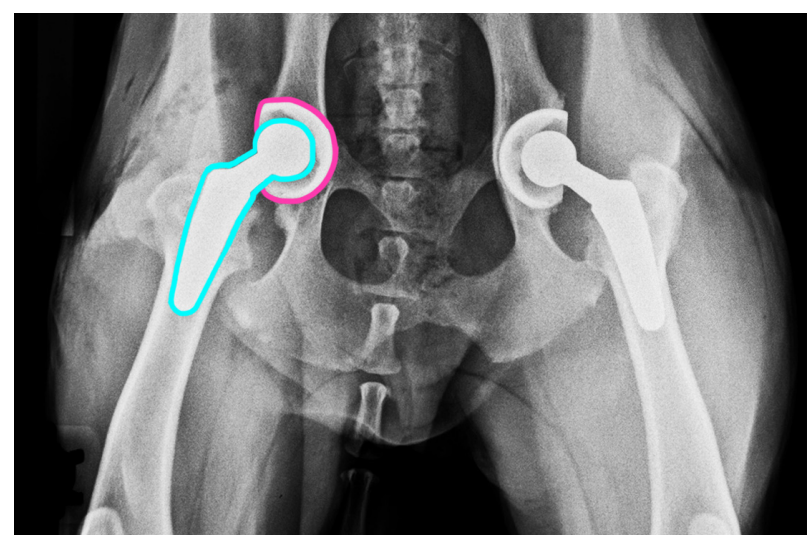

Figure 5 Radiograph illustrating a bilateral total hip replacement.

Notes: Blue line indicates femoral implant, pink line indicates acetabular implant. Image courtesy of Dr Jeffrey D Brourman. 
to implant particulate wear debris from aseptic implant loosening. ${ }^{83}$ Another consideration is that the persistence of joint laxity may influence the outcomes of total hip replacement. ${ }^{84}$ Efforts continue to improve upon available total hip replacement implants for dogs. A hybrid system of a cementless acetabular cup and a cemented femoral implant has been successfully applied in dogs relatively recently. ${ }^{85}$ Hip resurfacing to replace only joint surfaces versus the entire joint ${ }^{86}$ in dogs is under development, but clinical trials have yet to be reported. ${ }^{81}$ Unfortunately, large-scale, prospective randomized studies have yet to be conducted for comparison of long-term outcomes for various surgical procedures and nonsurgical management. Hence, individualized care remains largely based on clinician preference and experience. ${ }^{71}$

\section{Genetics}

While diagnosis and treatment of CHD are central to individual patient care, prevention by selective breeding will help obviate the presence of a debilitating condition in canine companions. With this in mind, there has been significant effort focused on identifying specific genes, genetic mutations, and quantitative trait loci (regions of chromosomes containing DNA for a specific trait), to use in conjunction with standard imaging methods for identification of CHD carriers. ${ }^{87,88}$ Genetic screening programs are complicated by the polygenic nature of $\mathrm{CHD}$ and related $\mathrm{OA}$, as well as environmental influences on phenotypic expression. A few promising quantitative trait loci for $\mathrm{OA}$ associated with $\mathrm{CHD}^{89}$ and the CHD phenotype in German shepherds ${ }^{90}$ have been identified relatively recently. Additionally, several chromosomal markers for CHD have been reported for a population of cross-bred Labrador Retriever-Greyhounds. ${ }^{91}$ Notably, several specific single nucleotide polymorphisms and positional candidate genes in dogs with CHD have been found to correlate with genes associated with the expression of OA and developmental dysplasia of the hip in humans. ${ }^{87}$ While the genomic underpinnings of CHD remain largely elusive, significant progress has been made and will continue, with expanding knowledge of the canine genome and interactions among genes that influence their expression.

\section{Future directions}

Despite almost a century of research, the complex etiology and optimal treatment paradigm for CHD remain elusive. As originally proposed by Schnelle at a meeting of the Veterinary Medical Society of New York City in the 1930s, ${ }^{92}$ CHD is not likely a single affliction but an array of heritable and environmentally induced degenerative disorders that differentially affect the morphology and function of the canine hip. The variable phenotypic expression of CHD makes development and implementation of standard identification procedures difficult. It may be possible to identify specific phenotypes within the broad spectrum of CHD similar to those of the human hip, like acetabular rim syndrome, acetabular retroversion, and femoral head necrosis. The relationship between articular damage in the human hip with morphology suggests a need to evaluate similar relationships in the dog. ${ }^{93}$ Identification and characterization of CHD phenotypes at the genetic, microstructural, and macrostructural levels will likely contribute to early detection and informed breeding decisions. Another area that will continue to promote progress in both imaging and treatment is development of novel measures on images obtained with contemporary imaging modalities like CT and magnetic resonance imaging. As evidence-based assessments of CHD prevention and treatment strategies become available, their selection and implementation will improve and facilitate the development of novel clinical approaches and surgical procedures. Incremental advances in the diagnosis, treatment, and prevention of the joint degeneration and pain associated with CHD through focused research and clinical evidence will continue to progress toward diminishing and eradicating CHD from our canine companions.

\section{Disclosure}

The authors report no conflicts of interest in this work.

\section{References}

1. Lust G. An overview of the pathogenesis of canine hip dysplasia. $J$ Am Vet Med Assoc. 1997;210:1443-1445.

2. Zhang ZW, Zhu L, Sandler J, et al. Estimation of heritabilities, genetic correlations, and breeding values of four traits that collectively define hip dysplasia in dogs. Am J Vet Res. 2009;70:483-492.

3. Schnelle GB. Bilateral congenital subluxation of the coxo-femoral joints in a dog. University of Pennsylvania Bulletin School of Veterinary Medicine Veterinary Extension Quarterly. 1937;37:15-16.

4. Impellizeri JA, Tetrick MA, Muir P. Effect of weight reduction on clinical signs of lameness in dogs with hip osteoarthritis. J Am Vet Med Assoc. 2001;216:1089-1091.

5. Kealy RD, Lawler DF, Ballam JM, et al. Evaluation of the effect of limited food consumption on radiographic evidence of osteoarthritis in dogs. J Am Vet Med Assoc. 2000;217:1678-1680.

6. Steinetz BG, Williams AJ, Lust G, Schwabe C, Büllesbach EE, Goldsmith LT. Transmission of relaxin and estrogens to suckling canine pups via milk and possible association with hip joint laxity. Am J Vet Res. 2008;69:59-67.

7. Greene LM, Marcellin-Little DJ, Lascelles BD. Associations among exercise duration, lameness severity, and hip joint range of motion in Labrador retrievers with hip dysplasia. J Am Vet Med Assoc. 2013;242: 1528-1533.

8. Madsen JS, Reimann I, Svalastoga E. Delayed ossification of the femoral head in dogs with hip dysplasia. J Small Anim Pract. 1991;32: 351-354.

9. Todhunter RJ, Zachos TA, Gilbert RO, et al. Onset of epiphyseal mineralization and growth plate closure in radiographically normal and dysplastic Labrador retrievers. J Am Vet Med Assoc. 1997;210: 1458-1462. 
10. The Orthopedic Foundation for Animals [webpage on the Internet]. The OFA's Hip Radiograph Procedures. Available from: http://www. offa.org/hd_procedures.html. Accessed January 10, 2015.

11. Roberts T, McGreevy PD. Selection for breed-specific long-bodied phenotypes is associated with increased expression of canine hip dysplasia. Vet J. 2010;183:266-272.

12. Farquhar T, Bertram J, Todhunter RJ, Burton-Warster N, Lust G. Variations in composition of cartilage from shoulder joints of young dogs at risk for developing canine hip dysplasia. J Am Vet Med Assoc. 1997;210:1483-1485.

13. Olsewski JM, Lust G, Rendano VT, Summers BA. Degenerative joint disease: multiple joint involvement in young and mature dogs. Am J Vet Res. 1983;44:1300-1308.

14. Leppänen M, Mäki K, Juga J, Saloniemi H. Factors affecting hip dysplasia in German shepherd dogs in Finland: efficacy of the current improvement programme. J Small Anim Pract. 2000;41:19-23.

15. Todhunter RJ, Lust G. Hip dysplasia: pathogenesis. In: Slatter ED, editor. Textbook of Small Animal Surgery. Philadelphia, PA, USA: Saunders; 2003.

16. Vanden Berg-Foels WS, Todhunter RJ, Schwager SJ, Reeves AP. Effect of early postnatal body weight on femoral head ossification onset and hip osteoarthritis in a canine model of developmental dysplasia of the hip. Pediatr Res. 2006;60:549-554.

17. Fujiki M, Kurima Y, Yamanokuchi K, Misumi K, Sakamoto H. Computed tomographic evaluation of growth-related changes in the hip joints of young dogs. Am J Vet Res. 2007;68:730-734.

18. Riser WH. The dysplastic hip joint: radiologic and histologic development. Vet Pathol. 1975;12:279-305.

19. Wu FM, Hedhammar $\AA$, Krook L. Overnutrition and skeletal disease. An experimental study in growing Great Dane dogs. IX. The long bones. Cornell Vet. 1974;64:83-114.

20. Chalmers HJ, Dykes NL, Lust G, et al. Assessment of bone mineral density of the femoral head in dogs with early osteoarthritis. Am J Vet Res. 2006;67:796-800.

21. Barr ARS, Benny HR, Gibbs C. Clinical hip dysplasia in growing dogs: the long-term results of conservative management. J Small Anim Pract. 1987;28:243-252.

22. Bijlsma JW, Berenbaum F, Lafeber FP. Osteoarthritis: an update with relevance for clinical practice. Lancet. 2011;377:2115-2126.

23. Buckwalter JA, Martin JA. Osteoarthritis. Adv Drug Deliv Rev. 2006;58: 150-167.

24. Grant ME, Prockop DJ. The biosynthesis of collagen. $N$ Engl J Med. 1972;286:194-199.

25. Skirving AP, Sims TJ, Bailey AJ. Congenital dislocation of the hip: a possible inborn error of collagen metabolism. J Inherit Metab Dis. $1984 ; 7: 27-31$

26. Madsen JS. The joint capsule and joint laxity in dogs with hip dysplasia. $J$ Am Vet Med Assoc. 1997;210:1463-1465.

27. Fry TR, Clark DM. Canine hip dysplasia: clinical signs and physical diagnosis. Vet Clin North Am Small Anim Pract. 1992;22:551-558.

28. Chalman JA, Butler HC. Coxofemoral joint laxity and the Ortolani sign. J Am Anim Hosp Assoc. 1985;21:671-676.

29. Ginja MMD, Silvestre AM, Gonzalo-Orden JM, Ferreira AJ. Diagnosis, genetic control and preventive management of canine hip dysplasia: a review. Vet J. 2010;184:269-276.

30. Puerto DA, Smith GK, Gregor TP, et al. Relationship between results of the Ortolani method of hip joint palpation and distraction index, Norberg angle, and hip score in dogs. J Am Vet Med Assoc. 1999;214: 497-501.

31. Bardens JW, Hardwick H. New observations on the diagnosis and cause of hip dysplasia. Vet Med Small Anim Clin. 1968;63:238-245.

32. Risler A, Klauer JM, Keuler NS, Adams WM. Puppy line, metaphyseal sclerosis, and caudolateral curvilinear and circumferential femoral head osteophytes in early detection of canine hip dysplasia. Vet Radiol Ultrasound. 2009;50:157-166.

33. Szabo SD, Biery DN, Lawler DF, et al. Evaluation of a circumferential femoral head osteophyte as an early indicator of osteoarthritis characteristic of canine hip dysplasia in dogs. J Am Vet Med Assoc. 2007;231: 889-892.
34. Gibbs C. The BVA/KC scoring scheme for control of hip dysplasia: interpretation of criteria. Vet Rec. 1997;141:275-284.

35. Dennis R. Interpretation and use of BVA/KC hip scores in dogs. In Practice. 2012;34:178-194.

36. Fédération Cynologique Internationale [webpage on the Internet]. FCI Scientific Commission. Available from: http://www.fci.be/en/FCIScientific-Commission-71.html. Accessed January 10, 2015.

37. Comhaire FH, Criel ACC, Dassy CAA, Guévar PGJ, Snaps FR. Precision, reproducibility, and clinical usefulness of measuring the Norberg angle by means of computerized image analysis. Am J Vet Res. 2009; 70:228-235.

38. Smith GK, Gregor TP, Rhodes WH, Biery DN. Coxofemoral joint laxity from distraction radiography and its contemporaneous and prospective correlation with laxity, subjective score and evidence of degenerative joint disease from conventional hip-extended radiography. Am JVet Res. 1993;54:1021-1042.

39. Smith GK, Biery DN, Gregor TP. New concepts of coxofemoral joint stability and development of a clinical stress-radiographic method for quantitating hip joint laxity in the dog. J Am Vet Med Assoc. 1990;196: $59-70$.

40. Lust G, Williams A, Burton-Warster N, et al. Joint laxity and its association with hip dysplasia in Labrador retrievers. Am J Vet Res. 1993;54: 1990-1999.

41. PennHIP [webpage on the Internet]. PennHIP Method: Measuring Hip Joint Laxity. http://info.antechimagingservices.com/pennhip/ navigation/penn-HIP-method/distraction-index-measuring-laxity.html. Accessed January 10, 2015.

42. Lopez MJ, Lewis BP, Swaab ME, Markel MD. Relationships among measurements obtained by use of computed tomography and radiography and scores of cartilage and microdamage in hip joints with moderate to severe joint laxity of adult dogs. Am J Vet Res. 2008;69:362-370.

43. Farese JP, Todhunter RJ, Lust G, Williams AJ, Dykes NL. Dorsolateral subluxation of hip joints in dogs measured in a weight-bearing position with radiography and computed tomography. Vet Surg. 1998;27: 393-405.

44. Malm S, Strandberg E, Danell B, Audell L, Swenson L, Hedhammar Å. Impact of sedation method on the diagnosis of hip and elbow dysplasia in Swedish dogs. Prev Vet Med. 2007;78:196-209.

45. Powers MY, Karbe GT, Gregor TP, et al. Evaluation of the relationship between Orthopedic Foundation for Animals' hip joint scores and PennHIP distraction index values in dogs. $J$ Am Vet Med Assoc. 2010;237:532-541

46. D'Amico LL, Xie L, Abell LK, Brown KT, Lopez MJ. Relationships of hip joint volume ratios with degrees of joint laxity and degenerative disease from youth to maturity in a canine population predisposed to hip joint osteoarthritis. Am J Vet Res. 2011;72:376-383.

47. Andronescu AA, Kelly L, Kearney MT, Lopez MJ. Associations between early radiographic and computed tomographic measures and canine hip joint osteoarthritis at maturity. Am J Vet Res. 2015;76:19-27.

48. Ginja MMD, Ferreira AJ, Jesus SS, et al. Comparison of clinical, radiographic, computed tomographic and magnetic resonance imaging methods for early prediction of canine hip laxity and dysplasia. Vet Radiol Ultrasound. 2009;50:135-143.

49. Preidler KW, Resnick D. Imaging of osteoarthritis. Radiol Clin North Am. 1996;34:259-271.

50. Hielm-Björkman AK, Kuusela E, Liman A, et al. Evaluation of methods for assessment of pain associated with chronic osteoarthritis in dogs. J Am Vet Med Assoc. 2003;222:1552-1558.

51. Kirkby KA, Lewis DD. Canine hip dysplasia: reviewing the evidence for nonsurgical management. Vet Surg. 2012;41:2-9.

52. Pollmeier M, Toulemonde C, Fleishman C, Hanson PD. Clinical evaluation of firocoxib and carprofen for the treatment of dogs with osteoarthritis. Vet Rec. 2006;159:547-551.

53. Smith GK, Paster ER, Powers MY, et al. Lifelong diet restriction and radiographic evidence of osteoarthritis of the hip joint in dogs. $J \mathrm{Am}$ Vet Med Assoc. 2006;229:690-693.

54. Rialland P, Bichot S, Lussier B, et al. Effect of a diet enriched with green-lipped mussel on pain behavior and functioning in dogs with clinical osteoarthritis. Can J Vet Res. 2013;77:66-74. 
55. Hielm-Björkman A, Roine J, Elo K, Lappalainen A, Junnila J, LaitinenVapaavuori O. An un-commissioned randomized, placebo-controlled double-blind study to test the effect of deep sea fish oil as a pain reliever for dogs suffering from canine OA. BMC Vet Res. 2012;8:157.

56. Fujiki M, Shineha J, Yamanokuchi K, Misumi K, Sakamoto H. Effects of treatment with polysulfated glycosaminoglycan on serum cartilage oligomeric matrix protein and C-reactive protein concentrations, serum matrix metalloproteinase- 2 and -9 activities, and lameness in dogs with osteoarthritis. Am J Vet Res. 2007;68:827-833.

57. Lust G, Williams AJ, Burton-Warster N, Beck KA, Rubin G. Effects of intramuscular administration of glycosaminoglycan polysulfates on signs of incipient hip dysplasia in growing pups. Am JVet Res. 1992;53: 1836-1843.

58. Todhunter RJ, Lust G. Polysulfated glycosaminoglycan in the treatment of osteoarthritis. J Am Vet Med Assoc. 1994;204:1245-1251.

59. White GW, Fregin GF, Selden JR. Effect of prophylactic intramuscular administration of polysulfated glycosaminoglycan on developmental and traumatic joint injuries in thoroughbred foals. $J$ Equine Vet Sci. 2007;27:107-111.

60. Durkes TE. Gold bead implants. Probl Ved Med. 1992;4:207-211.

61. Jaeger GT, Larsen S, Søli N, Moe L. Double-blind, placebo-controlled trial of the pain-relieving effects of the implantation of gold beads into dogs with hip dysplasia. Vet Rec. 2006;158:722-726.

62. Jaeger GT, Larsen S, Søli N, Moe L. Two years follow-up study of the pain-relieving effect of gold bead implantation in dogs with hip-joint arthritis. Acta Vet Scand. 2007;49:9.

63. Bollinger C, Decamp CE, Stajich M, et al. Gait analysis of dogs with hip dysplasia treated with gold bead implantation acupuncture. Vet Comp Orthop Traumatol. 2002;15:116-122.

64. Mlacnik E, Bockstahler BA, Müller M, Tetrick MA, Nap RC, Zentek J. Effects of caloric restriction and a moderate or intense physiotherapy program for treatment of lameness in overweight dogs with osteoarthritis. J Am Vet Med Assoc. 2006;229:1756-1760.

65. Heikklä HM, Hielm-Björkman AK, Morelius M, et al. Intra-articular botulinum toxin A for the treatment of osteoarthritic joint pain in dogs: a randomized, double-blinded, placebo-controlled clinical trial. Vet J. 2014;200:162-169.

66. Farrell M, Clements DN, Mellor D, et al. Retrospective evaluation of the long-term outcome of non-surgical management of 74 dogs with clinical hip dysplasia. Vet Rec. 2007;160:506-511.

67. Marx C, Silverira MD, Selbach I, et al. Acupoint injection of autologous stromal vascular fraction and allogeneic adipose-derived stem cells to treat hip dysplasia in dogs. Stem Cells Int. 2014;2014:391274.

68. Vilar JM, Batista M, Morales M, et al. Assessment of the effect of intraarticular injection of autologous adipose-derived mesenchymal stem cells in osteoarthritic dogs using a double blinded force platform analysis. BMC Vet Res. 2014;10:143.

69. Vilar JM, Morales M, Santana A, et al. Controlled, blinded force platform analysis of the effect of intraarticular injection of autologous adipose-derived mesenchymal stem cells associated to PRGF-Endoret in osteoarthritic dogs. BMC Vet Res. 2013;9:131.

70. Cuervo B, Rubio M, Sopena J, et al. Hip osteoarthritis in dogs: a randomized study using mesenchymal stem cells from adipose tissue and plasma rich in growth factors. Int J Mol Sci. 2014;15: 13437-13460.

71. Bergh MS, Budsberg SC. A systematic review of the literature describing the efficacy of surgical treatments for canine hip dysplasia. Vet Surg. 2014;43:501-506.

72. Manley PA, Adams WM, Danielson KC, Dueland RT, Linn KA. Long-term outcome of juvenile pubic symphysiodesis and triple pelvic osteotomy in dogs with hip dysplasia. J Am Vet Med Assoc. 2007;230: 206-210
73. Dueland RT, Adams WM, Patricelli AJ, Linn KA, Crump PM. Canine hip dysplasia treated by juvenile pubic symphysiodesis. Part I: two year results of computed tomography and distraction index. Vet Comp Orthop Traumatol. 2010;23:306-317.

74. Dueland RT, Adams WM, Fialkowski JP, Patricelli AJ, Mathews KG, Nordheim EV. Effects of pubic symphysiodesis in dysplastic puppies. Vet Surg. 2001;30:201-217.

75. Patricelli AJ, Dueland RT, Adams WM, Fialkowski JP, Linn KA, Nordheim EV. Juvenile pubic symphysiodesis in dysplastic puppies at 15 and 20 weeks of age. Vet Surg. 2002;31:435-444.

76. Slocum B, Devine T. Pelvic osteotomy technique for axial rotation of the acetabular segment in dogs. J Am Anim Hosp Assoc. 1986;22: 331-338.

77. Borostyankoi F, Rooks RL, Kobluk CN, Reed AL, Littledike ET. Results of single-session bilateral triple pelvic osteotomy with an eight-hole iliac bone plate in dogs: 95 cases (1996-1999). JAm Vet Med Assoc. 2003;222: 54-59.

78. Rose SA, Bruecker KA, Petersen SW, Uddin N. Use of locking plate and screws for triple pelvic osteotomy. Vet Surg. 2012;41:114-120.

79. Fitzpatrick N, Law AY, Bielecki MB, Girling S. Cementless total hip replacement in 20 juveniles using BFX ${ }^{\mathrm{TM}}$ arthroplasty. Vet Surg. 2014;43:715-725.

80. Lockwood AA, Liska WD. Duration of clinical signs prior to total hip replacement in dogs. J Am Vet Med Assoc. 2011;238:905-908.

81. Allen MJ. Advances in total joint replacement in small animals. J Small Anim Pract. 2012;53:495-506.

82. Morshed S, Bozic KJ, Ries MD, Malchau H, Colford JM. Comparison of cemented and uncemented fixation in total hip replacement. Acta Orthop. 2007; 78:315-326.

83. Harris WH. Wear and periprosthetic osteolysis: the problem. Clin Orthop Relat Res. 2001;393:66-70.

84. Hayes GM, Ramirez J, Hobbs JL. Does the degree of preoperative subluxation or soft tissue tension affect the incidence of postoperative luxation in dogs after total hip replacement? Vet Surg. 2009;40:6-13.

85. Gemmill TJ, Pink J, Renwick A, et al. Hybrid cemented/cementless total hip replacement in dogs: seventy-eight consecutive joint replacements. Vet Surg. 2010;40:621-630.

86. Bozic KJ, Pui CM, Ludeman MJ, Vail TP, Silverstein MD. Do the potential benefits of metal-on-metal hip resurfacing justify the increased cost and risk of complications? Clin Orthop Relat Res. 2010;468: 2301-2312.

87. Zhou Z, Sheng X, Zhang Z, et al. Differential genetic regulation of canine hip dysplasia and osteoarthritis. PLoS One. 2010;5:e13219.

88. Zhu L, Zhang Z, Friedenberg SS, et al. The long (and winding) road to gene discovery for canine hip dysplasia. Vet J. 2009;181:97-110.

89. Mateescu RG, Burton-Warster N, Tsai K, Phavaphutanon J, Zhang Z, Murphy KE. Identification of quantitative trait loci for osteoarthritis of hip joints in dogs. Am J Vet Res. 2008;69:1294-1300.

90. Fels L, Distl O. Identification and validation of quantitative trait loci (QTL) for canine hip dysplasia (CHD) in German shepherd dogs. PLoS One. 2014;9:e96618.

91. Todhunter RJ, Mateescu RG, Lust G, et al. Quantitative trait loci for hip dysplasia in a cross breed canine pedigree. Mamm Genome. 2005;16: 720-730.

92. Noble H. What radiology does for dogs: through x-ray pictures, veterinarians have been able to catalogue canine ills. American Kennel Gazette. 1935;1935:25-27.

93. Kaya M, Suzuki T, Emori M, Yamashita T. Hip morphology influences the pattern of articular cartilage damage. Knee Surg Sports Traumatol Arthrosc. September 11, 2014. [Epub ahead of print.] 


\section{Publish your work in this journal}

Veterinary Medicine: Research and Reports is an international, Visit http://www.dovepress.com/testimonials.php to read real quotes peer-reviewed, open access journal publishing original research, case reports, editorials, reviews and commentaries on all areas of veterinary medicine. The manuscript management system is completely online and includes a very quick and fair peer-review system.

Submit your manuscript here: http://www.dovepress.com/veterinary-medicine-research-and-reports-journal 